\title{
Timing of Adjuvant Chemotherapy and Survival in Patients with Stage II/III Rectal Cancer"
}

\author{
Isac S. F. Lima ${ }^{1}$, Yutaka Yasui ${ }^{1}$, Andrew Scarfe ${ }^{2,3}$, Marcy Winget ${ }^{1,4 \#}$ \\ ${ }^{1}$ School of Public Health, University of Alberta, Edmonton, Canada; ${ }^{2}$ Alberta Health Services, Cancer Care, Cross Cancer Institute, \\ Edmonton, Canada; ${ }^{3}$ Department of Oncology, University of Alberta, Edmonton, Canada; ${ }^{4}$ Alberta Health Services, Cancer Care, \\ Community Oncology, Edmonton, Canada. \\ Email: ${ }^{*}$ marcy.winget@albertahealthservices.ca
}

Received September $11^{\text {th }}, 2012$; revised October $10^{\text {th }}, 2012$; accepted October $19^{\text {th }}, 2012$

\begin{abstract}
Background: Treatment guidelines in the 1990s established surgery followed by chemoradiotherapy as the standard treatment for stage II/III rectal cancer. Our aim was to investigate the association between the timing of adjuvant treatment and patient survival in practice and identify demographic/clinical factors associated with treatment patterns. Methods: All residents of Alberta diagnosed with stage II/III rectal adenocarcinoma in 2000-2005 who had surgery were included in the study. Demographic and clinical data were obtained from the Alberta Cancer Registry and linked to hospital data and socioeconomic data from the 2001 Canadian Census. Overall and cancer-specific hazard ratios of death were estimated using Cox proportional hazards models. Results: 1243 patients were included in the study; 636 (51\%) patients received treatment consistent with guidelines. Patients who received adjuvant chemotherapy $12-16$ weeks after surgery or more than 16 weeks/ did not receive it had a $43 \%$ and $58 \%$ higher risk of rectal cancer death, respectively, compared to those who received it within 8 weeks of surgery. Conclusion: Adjuvant chemotherapy for stage II/III rectal cancer should be initiated within 12 weeks after surgery to maximize treatment benefits. Efforts to increase the proportion of patients treated within 12 weeks after surgery are needed.
\end{abstract}

Keywords: Adjuvant Treatment; Health Services Research; Rectal Cancer; Survival; Timeliness of Care; Treatment Guidelines

\section{Introduction}

The National Institute of Health (NIH) Consensus Conference in 1990 established surgical resection of adenocarcinoma of the rectum followed by chemo-radiotherapy as the recommended treatment for patients diagnosed with stage II or III disease [1]. This guideline was based on the results of several large randomized controlled trials [2-4] and became the standard of care for patients with stage II/III rectal cancer in the United States. More recent studies have shown that pre-operative (neoadjuvant) chemoradiotherapy significantly reduces local recurrence rates [5-9] and improves disease-free survival among stage II/III rectal cancer patients $[9,10]$. The majority of the patients in the clinical trials initiated adjuvant treatment within 6 weeks after surgery; this may not be easy to achieve in practice due to post-operative complications and/or healthcare system limitations. The op-

\footnotetext{
${ }^{*}$ This research was made possible by grants received from the Canadian Institute for Health Research, Canadian Cancer Society, Alberta Cancer Foundation, Alberta Ingenuity Centre for Machine Learning, Alberta Innovates - Health Solutions, and Canada Research Chair Program.

\#Corresponding author.
}

timum timing for adjuvant treatment initiation for surgically resected stage II/III rectal patients, therefore, remains unclear.

In Alberta, Canada, the recommendation for patients diagnosed with stage II/III rectal adenocarcinoma is neoadjuvant radiation with or without chemotherapy and/or adjuvant chemotherapy with or without radiation; the minimum recommended treatment is, therefore, surgery plus adjuvant chemotherapy. Adjuvant chemotherapy should be initiated within twelve weeks of surgery. These guidelines are consistent with current ones from the National Comprehensive Cancer Network [11] and European Society for Medical Oncology [12].

The goals of this study are to: 1) Describe treatment patterns of patients diagnosed with stage II/III rectal cancer between 2000 and 2005; 2) Estimate the proportion of patients receiving adjuvant chemotherapy within 12 weeks after surgery; 3) Identify patient/clinical characteristics associated with receipt of adjuvant chemotherapy within 12 weeks after surgery; and 4) Investigate the association between the timing of adjuvant chemotherapy and survival of patients diagnosed with stage 
II/III rectal cancer.

\section{Methods}

\subsection{Study Population and Inclusion Criteria}

The province of Alberta has approximately 3 million residents; about two-thirds live in Edmonton or Calgary, the two largest cities. The province provides universal, publicly-funded health care system for its residents. Standard cancer treatments are free to patients as are associated visits to cancer facilities, including consultations with oncologists. Physicians and hospitals are legally required to report every cancer case they diagnose to the Alberta Cancer Registry, a member of the North American Association of Comprehensive Cancer Registries. The Alberta Cancer Registry, established in 1942, is routinely recognized for the high quality and completeness of its data [13].

All residents of Alberta diagnosed in 2000 to 2005 with stage II or III rectal adenocarcinoma (International Classification of Diseases for Oncology (ICD-O) [14] code c19 and C20) were identified from the Alberta Cancer Registry. Patients were excluded if they did not receive surgery, died within 16 weeks of their surgery, were diagnosed with another primary cancer 6 months prior or subsequent to their rectal cancer diagnosis, did not have histologically confirmed adenocarcinoma, or were treated outside of Alberta. Cancer staging was based on the TNM (Tumor, Node, and Metastasis) system from the American Joint Committee on Cancer (AJCC version 6) [15]. Local invasive tumors (T3-4) that have not spread to regional lymph nodes (N0) or distant metastatic sites (M0) were defined as stage II. Invasive tumors of any size (T1-4) that have spread to at least one regional lymph node (N1-2) but not to distant metastatic sites (M0) were defined as stage III.

\subsection{Data Sources}

Data were linked from four different data sources: Alberta Cancer Registry, Ambulatory Care Classification System (ACCS), Discharge Abstract Database (DAD), and the 2001 Canadian census. The Alberta Cancer Registry is responsible for recording and maintaining data on all cancer cases and cancer deaths in Alberta. Patient demographics, tumor histology and stage, postal code of residence at diagnosis, initial treatment modalities and start dates, and date of death, if deceased, were obtained from the Alberta Cancer Registry. Mortality data are updated monthly using provincial vital statistics; cause of death is verified by the Alberta Cancer Registry using the International Classification of Disease (ICD-10) coding rules [16].

The ACCS and DAD databases contain diagnosis and procedure codes on all outpatient and inpatient hospital visits, respectively, in the province of Alberta. All hospital visits that occurred in the year prior to the patient's cancer diagnosis were used to identify co-morbidities using an enhancement [17] to the Charlson Co-morbidity Index [18].

The 2001 Canadian census was used to obtain socioeconomic indicators at the neighborhood level (census dissemination areas) for each patient. Four variables were used as measures of the neighborhood socioeconomic status: 1) Median income; 2) Proportion of employment; 3) Proportion separated, divorced, or widowed; and 4) Proportion not graduated from high school.

\subsection{Statistical Analysis}

Descriptive statistics were calculated and Chi-square or Fisher's Exact tests, as appropriate, were used to assess associations between patient/clinical characteristics and treatment regimen received. Treatment was categorized as: "Adjuvant treatment only" if post-operative chemotherapy with or without radiotherapy was received without any pre-operative treatment; "Neoadjuvant treatment only" if radiotherapy with or without chemotherapy was received prior to surgery, without any post-operative treatment; "Neo + adjuvant treatment" if both neoadjuvant and adjuvant treatment, as defined above, were received; and "Other treatment" if curative surgery or surgery followed by radiation therapy was received.

Time from the date of surgery to the date of first adjuvant chemotherapy session was calculated and patients were categorized into the following four groups for statistical analysis: received adjuvant chemotherapy within 8 weeks after surgery; 8 - 12 weeks after surgery; 12 - 16 weeks after surgery; or no adjuvant chemotherapy within 16 weeks of surgery. This latter group includes patients who received one of: 1) Adjuvant chemotherapy more than 16 weeks after surgery; 2) Neoadjuvant treatment (chemotherapy and/or radiotherapy) plus surgery; 3) Surgery plus adjuvant radiotherapy but no adjuvant chemotherapy; or 4) Only surgery. The outer limit of 16 weeks for adjuvant chemotherapy was determined based on expected clinical practice and previous studies [19]. These categories were used to evaluate the association between timing of adjuvant chemotherapy initiation and patient survival.

Kaplan-Meier curves were used to describe patient survival stratified by time from surgery to adjuvant chemotherapy. Cumulative incidence curves were used to describe the cumulative mortality due to rectal cancerspecific deaths, treating other causes of death as competing risk [20]. The Kaplan-Meier and cumulative incidence curves were started at 16 weeks after surgery. Deaths prior to this starting time were not included in the 
analysis as this is the earliest time point that allows all "time from surgery to adjuvant chemotherapy" groups to be defined.

Cox proportional hazards models were used to estimate the adjusted hazard ratios (HRs) of overall and cancer-specific mortality by time from surgery to adjuvant chemotherapy (time dependent covariate), starting at 16 weeks after surgery. In order to closely adjust for age at diagnosis, a natural cubic spline of age at diagnosis was used with four knots [21]. Test for trends were performed for time to adjuvant chemotherapy and year of diagnosis; p-values were calculated adjusting for all variables in the model. Patients were followed to the first event of death or March 31, 2009.

All statistical analyses were conducted using SAS statistical software version 9.2 (SAS Institute, Cary, NC, USA) and R version 2.9 (R Foundation for Statistical Computing, Vienna, Austria).

\section{Results}

There were 1394 residents of Alberta diagnosed with stage II or III rectal cancer in the years 2000 to 2005 . The following number of patients were excluded from the study based on the exclusion criteria described above: 40 patients did not have surgery; 50 patients were diagnosed with another cancer within six months prior or subsequent to their rectal cancer diagnosis; 14 patients did not have histological confirmation of their disease; 6 patients had a histology other than adenocarcinoma; 1 patient was treated outside of Alberta; and 40 patients died within 16 weeks after surgery. The remaining 1243 patients were included in the study.

Table 1 shows the baseline characteristics of the 1243 patients included in the study stratified by type of treatment received. Overall, 473 (38\%) of the stage II/III rectal cancer patients had surgery only or surgery followed by radiotherapy without post-operative chemotherapy, $134(11 \%)$ received neoadjuvant treatment only, $501(40 \%)$ received adjuvant treatment only, and $135(11 \%)$ patients received both neoadjuvant and adjuvant treatment. The "neoadjuvant + adjuvant" and "adjuvant only" groups (51\%) include the patients who had treatment consistent with guidelines whereas the other two groups (49\%) include the patients who did not receive treatment consistent with treatment guidelines.

Treatment differed by stage ( $\mathrm{p}<0.001) ; 61 \%$ of patients diagnosed with stage II rectal cancer received incomplete treatment, $431(91 \%)$ received surgery only and the remaining $42(9 \%)$ patients received radiotherapy prior to surgery without chemotherapy. Conversely, $11 \%$ of the patients with stage III rectal cancer received both neoadjuvant treatment and adjuvant chemotherapy and $53 \%$ received adjuvant treatment that includes chemo- therapy only. Higher co-morbidity scores, older age, and living in lower socioeconomic neighborhoods were each associated with lower rates of both pre- and post-surgical treatment (Table 1).

Table 2 shows the demographic, clinical, and neighborhood-level socioeconomic characteristics of patients stratified by time from surgery to the receipt of adjuvant chemotherapy. Stage II rectal cancer patients, those aged 75 years and older, and those with two or more severe co-morbidities were least likely to receive adjuvant chemotherapy or receive it within 12 weeks of surgery. Lower rates of adjuvant chemotherapy were also seen among those who live in neighborhoods with a high percentage of divorced, separated or widowed, a low employment rate, low high school graduation rate, and/or a low median household income.

Figures 1 and $\mathbf{2}$ show the Kaplan-Meier and cumulative incidence curves for the overall survival and rectal cancer-specific mortality, respectively, by time from surgery to adjuvant chemotherapy. In Figure 2, the rectal cancer-specific mortality appears to be grouped into two groups: 1) Those who did not receive adjuvant chemotherapy or received it 12 weeks or more after surgery; and 2) Those who received it within 12 weeks. Those who received adjuvant chemotherapy within 12 weeks of their surgery had a considerably lower rectal cancerspecific mortality compared to those who did not receive it (Figure 2).

Table 3 presents the fit of the Cox proportional hazards models. The adjusted mortality HRs and corresponding 95\% confidence intervals (overall and rectal cancerspecific) for patients with stage II/III rectal cancer were adjusted for all variables shown in the table. There was no difference in the overall or rectal cancer-specific mortality hazard for patients who received adjuvant chemotherapy $8-12$ weeks after surgery relative to those who received it within 8 weeks. The rectal cancer-specific HR for patients who received adjuvant chemotherapy 12 - 16 weeks after surgery, compared to those who received it within 8 weeks after surgery, was 1.43 (95\% confidence interval (CI) $0.81-2.53$ ). This estimate was similar to the HR for those who received it 16 weeks after surgery or more or did not receive adjuvant chemotherapy, 1.58 (95\% CI 1.09 - 2.30). The same group of patients also had a 1.68 times higher overall mortality hazard compared to those who received chemotherapy within 8 weeks after surgery $(\mathrm{HR}=1.68,95 \%$ CI $1.22-2.32)$.

\section{Discussion}

The primary aim of this study was to determine whether there was a relationship in clinical practice between the timing of initiation of adjuvant chemotherapy and survival for patients diagnosed with stage II/III rectal cancer. 
Table 1. Characteristics of patients with stage II/III rectal cancer in Alberta with respect to treatments received.

\begin{tabular}{|c|c|c|c|c|c|c|c|c|c|c|}
\hline \multirow[b]{3}{*}{$\begin{array}{c}\text { Patients' } \\
\text { characteristics }\end{array}$} & & & \multicolumn{8}{|c|}{ Treatments $N\left(\%^{\mathrm{a}}\right)$} \\
\hline & & & \multicolumn{4}{|c|}{ Guideline-adherent } & \multicolumn{4}{|c|}{ Non-adherent } \\
\hline & \multicolumn{2}{|c|}{$\begin{array}{c}\text { Total } \\
N\left(\%{ }^{b}\right)\end{array}$} & \multicolumn{2}{|c|}{$\begin{array}{c}\text { Neo + adjuvant } \\
\text { treatment }\end{array}$} & \multicolumn{2}{|c|}{$\begin{array}{c}\text { Adjuvant treatment } \\
\text { only }\end{array}$} & \multicolumn{2}{|c|}{$\begin{array}{c}\text { Neoadjuvant treatment } \\
\text { only }\end{array}$} & \multicolumn{2}{|c|}{ Other treatment } \\
\hline Total & 1243 & (100) & 135 & (11) & 501 & (40) & 134 & (11) & 473 & (38) \\
\hline \multicolumn{11}{|l|}{ Time to adjuvant chemotherapy ${ }^{* * *}$} \\
\hline$<8$ weeks & 244 & $(20)$ & 54 & $(22)$ & 190 & $(78)$ & 0 & $(0)$ & 0 & $(0)$ \\
\hline $8-12$ weeks & 290 & $(23)$ & 62 & $(21)$ & 228 & $(79)$ & 0 & $(0)$ & 0 & $(0)$ \\
\hline $12-16$ weeks & 71 & $(6)$ & 13 & $(18)$ & 58 & $(82)$ & 0 & $(0)$ & 0 & $(0)$ \\
\hline $16+$ weeks/no adjuvant chemo & 638 & $(51)$ & 6 & (1) & 25 & (4) & 134 & $(21)$ & 473 & $(74)$ \\
\hline \multicolumn{11}{|l|}{ Stage $^{* * *}$} \\
\hline II & 618 & $(50)$ & 69 & $(11)$ & 167 & $(27)$ & 89 & (14) & 293 & $(47)$ \\
\hline III & 625 & $(50)$ & 66 & $(11)$ & 334 & $(53)$ & 45 & (7) & 180 & $(29)$ \\
\hline \multicolumn{11}{|l|}{$\operatorname{Sex}^{* *}$} \\
\hline Female & 475 & $(38)$ & 43 & (9) & 188 & $(40)$ & 37 & (8) & 207 & (44) \\
\hline Male & 768 & $(62)$ & 92 & $(12)$ & 313 & $(41)$ & 97 & (13) & 266 & $(35)$ \\
\hline \multicolumn{11}{|l|}{ Age at diagnosis ${ }^{* * *}$} \\
\hline$<65$ years & 516 & $(42)$ & 91 & $(18)$ & 283 & $(55)$ & 74 & (14) & 68 & (13) \\
\hline $65-75$ years & 374 & $(30)$ & 31 & (8) & 177 & $(47)$ & 35 & (9) & 131 & $(35)$ \\
\hline$\geq 75$ years & 353 & $(28)$ & 13 & (4) & 41 & $(12)$ & 25 & (7) & 274 & $(78)$ \\
\hline \multicolumn{11}{|l|}{ Residence at diagnosis $^{* *}$} \\
\hline South & 108 & (9) & 10 & (9) & 44 & $(41)$ & 6 & $(6)$ & 48 & $(44)$ \\
\hline Calgary \& area & 408 & $(33)$ & 41 & $(10)$ & 182 & $(45)$ & 40 & $(10)$ & 145 & $(36)$ \\
\hline Central & 193 & $(16)$ & 14 & (7) & 72 & $(37)$ & 15 & $(8)$ & 92 & $(48)$ \\
\hline Edmonton \& area & 398 & $(32)$ & 52 & $(13)$ & 145 & $(36)$ & 56 & $(14)$ & 145 & $(36)$ \\
\hline North & 136 & $(11)$ & 18 & $(13)$ & 58 & $(43)$ & 17 & $(13)$ & 43 & $(32)$ \\
\hline \multicolumn{11}{|c|}{$\%$ Divorced, separated or widowed ${ }^{* * * *}$} \\
\hline$<13 \% \mathrm{~d} / \mathrm{s} / \mathrm{w}$ & 385 & $(32)$ & 47 & (12) & 174 & $(45)$ & 51 & (13) & 113 & $(29)$ \\
\hline $13 \%-29 \% \mathrm{~d} / \mathrm{s} / \mathrm{w}$ & 712 & $(59)$ & 76 & (11) & 284 & $(40)$ & 71 & $(10)$ & 281 & (39) \\
\hline$\geq 29 \% \mathrm{~d} / \mathrm{s} / \mathrm{w}$ & 109 & (9) & 9 & (8) & 29 & (27) & 10 & (9) & 61 & $(56)$ \\
\hline \multicolumn{11}{|l|}{ \% Employed ${ }^{* * *}$} \\
\hline$<60 \%$ & 350 & (29) & 35 & $(10)$ & 116 & (33) & 40 & (11) & 159 & $(45)$ \\
\hline $60 \%-71 \%$ & 432 & $(36)$ & 47 & (11) & 171 & $(40)$ & 45 & $(10)$ & 169 & (39) \\
\hline$\geq 71 \%$ & 424 & (35) & 50 & (12) & 200 & (47) & 47 & (11) & 127 & (30) \\
\hline \multicolumn{11}{|l|}{ Median annual household income ${ }^{* * *}$} \\
\hline$(\mathrm{Q} 1)<38,885$ & 291 & $(24)$ & 34 & $(12)$ & 91 & $(31)$ & 32 & $(11)$ & 134 & $(46)$ \\
\hline (Q2) $38,885-51,004$ & 318 & $(26)$ & 28 & (9) & 129 & $(41)$ & 40 & (13) & 121 & $(38)$ \\
\hline (Q3) $51,004-66,774$ & 316 & $(26)$ & 34 & $(11)$ & 129 & $(41)$ & 28 & (9) & 125 & $(40)$ \\
\hline (Q4) 66,774 or more & 281 & $(23)$ & 36 & $(13)$ & 138 & $(49)$ & 32 & $(11)$ & 75 & $(27)$ \\
\hline \multicolumn{11}{|c|}{$\%$ Not graduated from high school ${ }^{* * *}$} \\
\hline$<27 \%($ median $)$ & 586 & $(49)$ & 69 & $(12)$ & 267 & $(46)$ & 68 & $(12)$ & 182 & $(31)$ \\
\hline$\geq 27 \%$ (median) & 620 & $(51)$ & 63 & $(10)$ & 220 & $(35)$ & 64 & $(10)$ & 273 & $(44)$ \\
\hline No. of co-morbidities ${ }^{* * *}$ & & & & & & & & & & \\
\hline 0 & 1043 & $(84)$ & 131 & (13) & 451 & $(43)$ & 114 & (11) & 347 & (33) \\
\hline 1 & 134 & $(11)$ & 2 & (1) & 39 & $(29)$ & 13 & $(10)$ & 80 & $(60)$ \\
\hline 2 or more & 66 & (5) & 2 & (3) & 11 & (17) & 7 & (11) & 46 & $(70)$ \\
\hline Year of diagnosis ${ }^{* * *}$ & & & & & & & & & & \\
\hline 2000 & 178 & (14) & 11 & (6) & 60 & $(34)$ & 14 & (8) & 93 & $(52)$ \\
\hline 2001 & 182 & $(15)$ & 6 & (3) & 71 & (39) & 28 & $(15)$ & 77 & $(42)$ \\
\hline 2002 & 194 & $(16)$ & 19 & $(10)$ & 89 & $(46)$ & 9 & $(5)$ & 77 & (40) \\
\hline 2003 & 208 & (17) & 16 & (8) & 99 & (48) & 17 & (8) & 76 & (37) \\
\hline 2004 & 227 & (18) & 29 & (13) & 84 & (37) & 33 & (15) & 81 & (36) \\
\hline 2005 & 254 & (20) & 54 & $(21)$ & 98 & (39) & 33 & (13) & 69 & (27) \\
\hline
\end{tabular}

${ }^{* * *} \mathrm{p}<0.001 ;{ }^{* *} \mathrm{p}<0.01 ;{ }^{*} \mathrm{p}<0.05 \mathrm{p}$-values are based on tests of equality across the four "treatment" groups and the categories of the corresponding variable. ${ }^{\mathrm{a}}$ Row percent; ${ }^{\mathrm{b}}$ Column percent; ${ }^{\mathrm{c}}$ Includes patients who received either surgery only or surgery followed by radiotherapy. 
Table 2. Characteristics of patients with stage II/III rectal cancer in Alberta with respect to timing of adjuvant chemotherapy.

Time to adjuvant chemotherapy $N\left(\%{ }^{a}\right)$

\begin{tabular}{|c|c|c|c|c|c|c|c|c|}
\hline \multirow{3}{*}{$\begin{array}{c}\text { Patient characteristics } \\
\text { Total } \\
\text { Stage }^{* * * *}\end{array}$} & \multicolumn{2}{|c|}{$\begin{array}{l}\text { Total } \\
N\left(\%^{b}\right)\end{array}$} & \multicolumn{2}{|c|}{$\leq 12$ weeks } & \multicolumn{2}{|c|}{12 - 16 weeks } & \multicolumn{2}{|c|}{$\begin{array}{c}16+\begin{array}{c}\text { weeks /no adjuvant } \\
\text { chemotherapy }\end{array} \\
\end{array}$} \\
\hline & 1243 & (100) & 534 & (43) & 71 & (6) & 638 & (51) \\
\hline & & & & & & & & \\
\hline II & 618 & $(50)$ & 189 & $(31)$ & 32 & (5) & 397 & $(64)$ \\
\hline III & 625 & $(50)$ & 345 & $(55)$ & 39 & (6) & 241 & (39) \\
\hline \multicolumn{9}{|l|}{ Sex } \\
\hline Female & 475 & $(38)$ & 189 & $(40)$ & 29 & (6) & 257 & $(54)$ \\
\hline Male & 768 & $(62)$ & 345 & $(45)$ & 42 & (5) & 381 & $(50)$ \\
\hline \multicolumn{9}{|l|}{ Age at diagnosis ${ }^{* * *}$} \\
\hline$<65$ years & 516 & $(42)$ & 326 & $(63)$ & 37 & (7) & 153 & $(30)$ \\
\hline $65-75$ years & 374 & $(30)$ & 167 & $(45)$ & 28 & (7) & 179 & $(48)$ \\
\hline$\geq 75$ years & 353 & $(28)$ & 41 & $(12)$ & 6 & $(2)$ & 306 & $(87)$ \\
\hline \multicolumn{9}{|l|}{ Residence at diagnosis ${ }^{* *}$} \\
\hline South & 108 & (9) & 47 & $(44)$ & 4 & (4) & 57 & $(53)$ \\
\hline Calgary \& area & 408 & $(33)$ & 173 & $(42)$ & 33 & $(8)$ & 202 & $(50)$ \\
\hline Central & 193 & $(16)$ & 65 & $(34)$ & 16 & $(8)$ & 112 & $(58)$ \\
\hline Edmonton \& area & 398 & $(32)$ & 184 & $(46)$ & 11 & (3) & 203 & $(51)$ \\
\hline North & 136 & $(11)$ & 65 & $(48)$ & 7 & $(5)$ & 64 & $(47)$ \\
\hline \multicolumn{9}{|c|}{ \% Divorced, separated or widowed ${ }^{* * *}$} \\
\hline$<13 \% \mathrm{~d} / \mathrm{s} / \mathrm{w}$ & 385 & $(32)$ & 183 & $(48)$ & 30 & $(8)$ & 172 & $(45)$ \\
\hline $13 \%-29 \% \mathrm{~d} / \mathrm{s} / \mathrm{w}$ & 712 & $(59)$ & 308 & $(43)$ & 33 & $(5)$ & 371 & $(52)$ \\
\hline$\geq 29 \% \mathrm{~d} / \mathrm{s} / \mathrm{w}$ & 109 & (9) & 31 & $(28)$ & 5 & $(5)$ & 73 & $(67)$ \\
\hline \multicolumn{9}{|l|}{ \% Employed ${ }^{* * *}$} \\
\hline$<60 \%$ & 350 & $(29)$ & 123 & $(35)$ & 18 & $(5)$ & 209 & $(60)$ \\
\hline $60 \%-71 \%$ & 432 & $(36)$ & 186 & $(43)$ & 21 & $(5)$ & 225 & $(52)$ \\
\hline$\geq 71 \%$ & 424 & $(35)$ & 213 & $(50)$ & 29 & (7) & 182 & $(43)$ \\
\hline \multicolumn{9}{|c|}{ Median annual household income ${ }^{* *}$} \\
\hline$(\mathrm{Q} 1)<38,885$ & 291 & $(24)$ & 106 & $(36)$ & 13 & (4) & 172 & $(59)$ \\
\hline (Q2) 38,885 - 51,004 & 318 & $(26)$ & 133 & $(42)$ & 19 & (6) & 166 & $(52)$ \\
\hline (Q3) 51,004 - 66,774 & 316 & $(26)$ & 139 & $(44)$ & 15 & $(5)$ & 162 & $(51)$ \\
\hline (Q4) 66,774 or more & 281 & $(23)$ & 144 & $(51)$ & 21 & (7) & 116 & $(41)$ \\
\hline \multicolumn{9}{|c|}{$\%$ Not graduated from high school ${ }^{* * * *}$} \\
\hline$<27 \%$ (median) & 586 & $(49)$ & 278 & $(47)$ & 40 & (7) & 268 & $(46)$ \\
\hline$\geq 27 \%$ (median) & 620 & $(51)$ & 244 & $(39)$ & 28 & $(5)$ & 348 & $(56)$ \\
\hline \multicolumn{9}{|l|}{ No. of co-morbidities ${ }^{* * *}$} \\
\hline 0 & 1043 & $(84)$ & 487 & $(47)$ & 67 & $(6)$ & 489 & $(47)$ \\
\hline 1 & 134 & $(11)$ & 36 & $(27)$ & 2 & $(1)$ & 96 & (72) \\
\hline 2 or more & 66 & (5) & 11 & $(17)$ & 2 & (3) & 53 & $(80)$ \\
\hline \multicolumn{9}{|l|}{ Year of diagnosis** } \\
\hline 2000 & 178 & $(14)$ & 62 & $(35)$ & 7 & (4) & 109 & $(61)$ \\
\hline 2001 & 182 & $(15)$ & 60 & (33) & 11 & (6) & 111 & $(61)$ \\
\hline 2002 & 194 & $(16)$ & 89 & $(46)$ & 14 & (7) & 91 & $(47)$ \\
\hline 2003 & 208 & $(17)$ & 96 & $(46)$ & 14 & (7) & 98 & (47) \\
\hline 2004 & 227 & $(18)$ & 95 & $(42)$ & 10 & (4) & 122 & $(54)$ \\
\hline 2005 & 254 & $(20)$ & 132 & $(52)$ & 15 & $(6)$ & 107 & $(42)$ \\
\hline
\end{tabular}

${ }^{* * *} \mathrm{p}<0.001 ;{ }^{* *} \mathrm{p}<0.01 ;{ }^{*} \mathrm{p}<0.05 \mathrm{p}$-values are based on tests of equality across the three "Time to Adjuvant Chemotherapy" groups and the categories of the corresponding variable. ${ }^{\mathrm{a}}$ Row percent; ${ }^{\mathrm{b}}$ Column percent. 


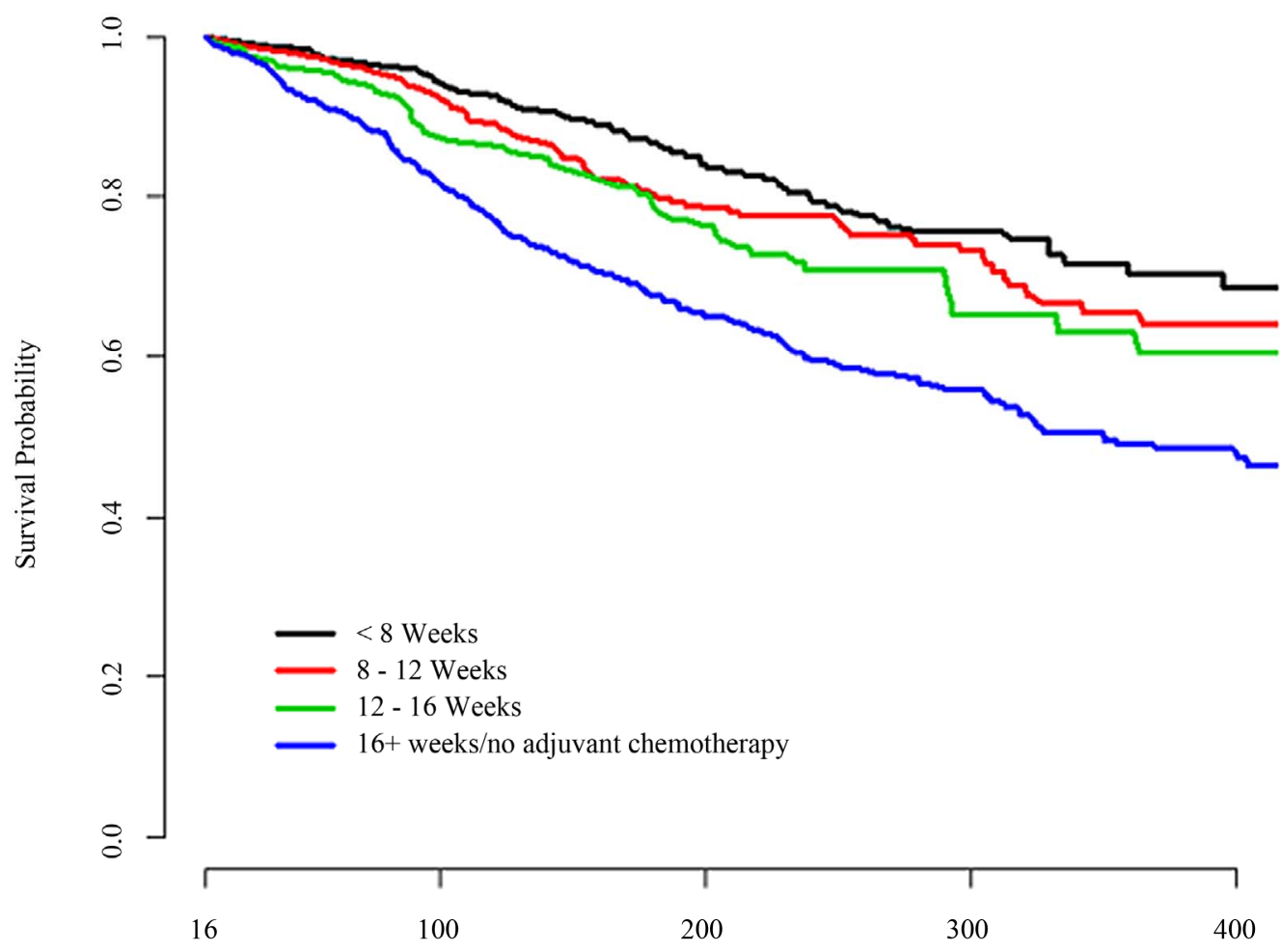

Time from surgery (weeks)

Figure 1. Kaplan-Meier survival curves for all-cause death.

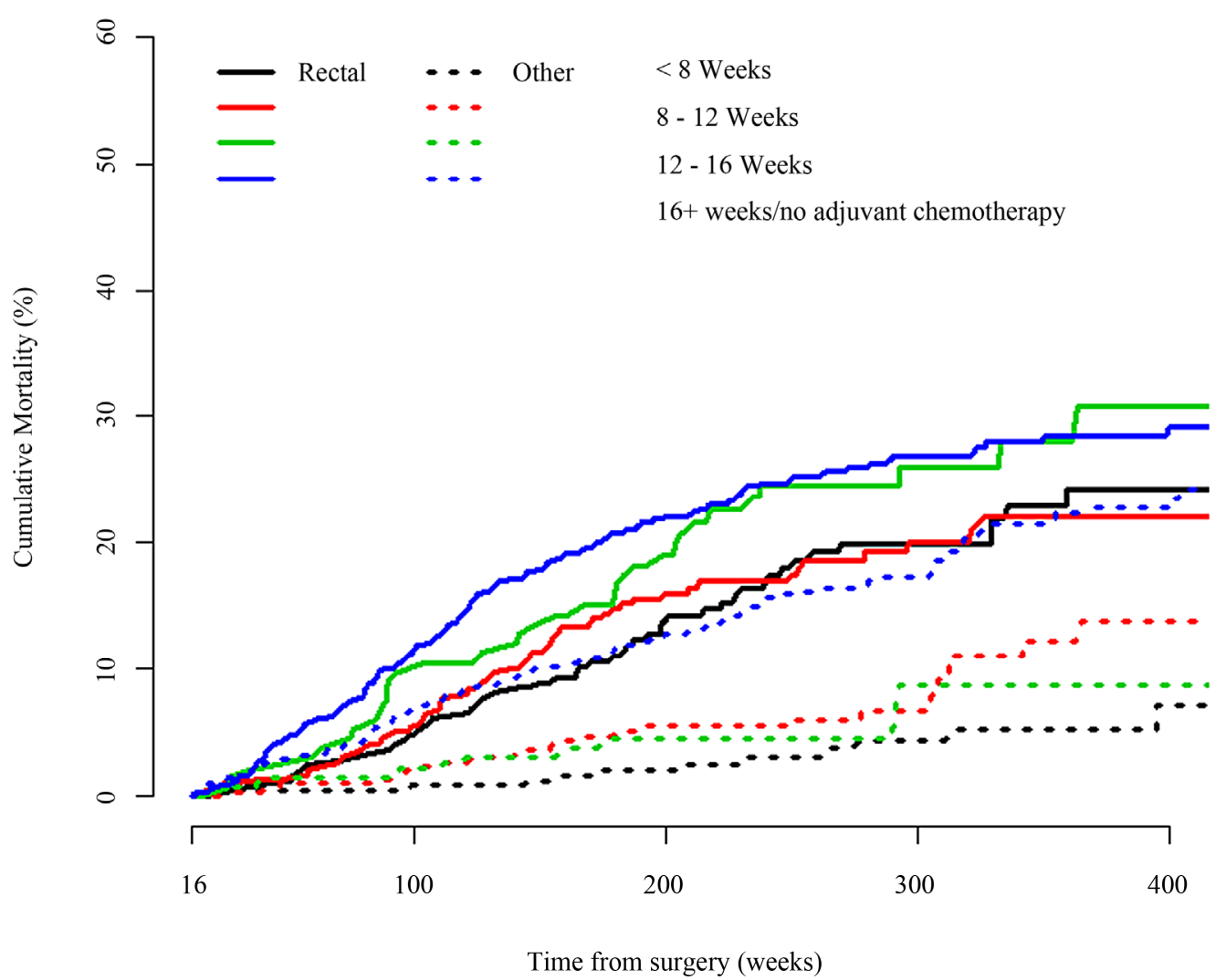

Figure 2. Cumulative cause-specific mortality by time to adjuvant treatment. 


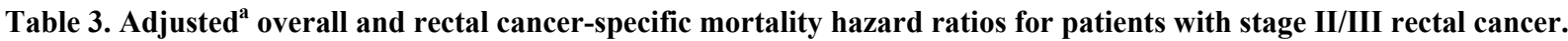

\begin{tabular}{|c|c|c|c|c|}
\hline \multirow{2}{*}{ Covariates } & \multicolumn{2}{|c|}{ Overall mortality $^{\mathrm{b}}$} & \multicolumn{2}{|c|}{ Rectal cancer mortality ${ }^{b}$} \\
\hline & $\begin{array}{c}\text { Adjusted hazard ratios } \\
(95 \% \mathrm{CI})\end{array}$ & p-value & $\begin{array}{c}\text { Adjusted hazard ratios } \\
\text { (95\% CI) }\end{array}$ & p-value \\
\hline Time to adjuvant chemotherapy & & $<0.001^{\mathrm{c}}$ & & $0.03^{\mathrm{c}}$ \\
\hline$<8$ weeks & ref & & ref & \\
\hline 8 - 12 weeks & $1.13(0.79-1.61)$ & 0.51 & $1.01(0.68-1.52)$ & 0.95 \\
\hline $12-16$ weeks & $1.29(0.76-2.20)$ & 0.35 & $1.43(0.81-2.53)$ & 0.22 \\
\hline $16+$ weeks/no adjuvant chemotherapy & $1.68(1.22-2.32)$ & 0.002 & $1.58(1.09-2.30)$ & 0.02 \\
\hline Neoadjuvant treatment & & 0.02 & & 0.01 \\
\hline No & ref & & ref & \\
\hline Yes & $1.39(1.05-1.84)$ & 0.02 & $1.52(1.09-2.11)$ & 0.01 \\
\hline Stage & & $<0.001$ & & $<0.001$ \\
\hline II & ref & & ref & \\
\hline III & $2.12(1.73-2.61)$ & $<0.001$ & $2.54(1.96-3.31)$ & $<0.001$ \\
\hline Sex & & 0.002 & & 0.71 \\
\hline Male & ref & & ref & \\
\hline Female & $0.72(0.58-0.89)$ & 0.002 & $0.95(0.74-1.23)$ & 0.72 \\
\hline Residence at diagnosis & & 0.32 & & 0.06 \\
\hline Edmonton \& area & ref & & ref & \\
\hline South & $1.13(0.79-1.63)$ & 0.50 & $1.64(1.06-2.54)$ & 0.03 \\
\hline Calgary \& area & $0.92(0.71-1.19)$ & 0.53 & $1.21(0.87-1.69)$ & 0.25 \\
\hline Central & $1.27(0.95-1.70)$ & 0.11 & $1.68(1.16-2.43)$ & 0.006 \\
\hline North & $1.17(0.82-1.68)$ & 0.38 & $1.42(0.91-2.21)$ & 0.13 \\
\hline \% Divorced, separated or widowed & & 0.18 & & 0.21 \\
\hline$<13 \% \mathrm{~d} / \mathrm{s} / \mathrm{w}$ & ref & & ref & \\
\hline $13 \%-29 \% \mathrm{~d} / \mathrm{s} / \mathrm{w}$ & $1.31(0.98-1.76)$ & 0.07 & $1.29(0.91-1.85)$ & 0.15 \\
\hline$\geq 29 \% \mathrm{~d} / \mathrm{s} / \mathrm{w}$ & $1.20(0.76-1.91)$ & 0.43 & $0.98(0.55-1.78)$ & 0.96 \\
\hline$\%$ Employed & & 0.27 & & 0.24 \\
\hline$<60 \%$ & $0.85(0.64-1.13)$ & 0.27 & $0.91(0.64-1.31)$ & 0.63 \\
\hline $60 \%-71 \%$ & $1.04(0.81-1.33)$ & 0.77 & $1.17(0.86-1.59)$ & 0.31 \\
\hline$\geq 71 \%$ & ref & & ref & \\
\hline Median annual household income & & 0.58 & & 0.66 \\
\hline$(\mathrm{Q} 1)<38,885$ & $0.94(0.62-1.42)$ & 0.76 & $1.09(0.65-1.83)$ & 0.75 \\
\hline (Q2) $38,885-51,004$ & $1.01(0.69-1.48)$ & 0.96 & $1.18(0.74-1.88)$ & 0.49 \\
\hline (Q3) 51,004 - 66,774 & $0.85(0.60-1.20)$ & 0.35 & $0.95(0.61-1.46)$ & 0.80 \\
\hline (Q4) 66,774 or more & ref & & ref & \\
\hline No. of co-morbidities & & $<0.007$ & & 0.54 \\
\hline 0 & ref & & ref & \\
\hline 1 & $1.23(0.93-1.63)$ & 0.14 & $1.18(0.82-1.71)$ & 0.36 \\
\hline 2 or more & $1.72(1.21-2.44)$ & 0.002 & $1.22(0.73-2.03)$ & 0.45 \\
\hline Year of diagnosis & & $<0.01^{\mathrm{c}}$ & & 0.98 \\
\hline 2000 & ref & & ref & \\
\hline 2001 & $0.84(0.61-1.16)$ & 0.30 & $0.98(0.66-1.47)$ & 0.93 \\
\hline 2002 & $0.80(0.58-1.12)$ & 0.19 & $0.94(0.61-1.43)$ & 0.75 \\
\hline 2003 & $0.68(0.48-0.98)$ & 0.04 & $0.90(0.57-1.41)$ & 0.63 \\
\hline 2004 & $0.81(0.57-1.15)$ & 0.23 & $1.05(0.67-1.65)$ & 0.82 \\
\hline 2005 & $0.63(0.42-0.94)$ & 0.02 & $0.93(0.56-1.54)$ & 0.77 \\
\hline
\end{tabular}

${ }^{a}$ Adjusted for all variables shown in the table plus age at diagnosis using a cubic spline with 4 knots and \% graduated from high school in the neighborhood. ${ }^{\mathrm{b}}$ Survival time starts at 16 weeks after surgery; ${ }^{\mathrm{c}}$ Test for trend. 
Additionally, we aimed to quantify the proportion of patients who received adjuvant chemotherapy within 12 weeks after surgery and their association with patient/ clinical characteristics.

Of those diagnosed with stage II/III rectal cancer in Alberta between 2000 and 2005 and who met the inclusion criteria, $534(43 \%)$ patients received adjuvant chemotherapy within 12 weeks after surgery. The remaining patients, however, either received delayed adjuvant chemotherapy or did not receive it. Factors strongly associated with not receiving adjuvant chemotherapy were: patient age 75 years or older; diagnosed with stage II disease; presence of one or more serious co-morbidities; living in neighborhoods with low socio-economic indicators; and region of residence. Both clinical trials and population-based studies have shown that elderly patients and those with co-morbidities can benefit from pre-operative $[6,9,10]$ and post-operative $[8,22-26]$ therapy. Older age and co-morbidities may be related to postsurgical complications or delayed recovery that could affect whether a patient received adjuvant chemotherapy and/or the timing of it; we were not able to evaluate these possibilities in our study.

In a publicly-funded healthcare system, however, factors such as region of residence and socioeconomic status should not be related to the receipt of standard treatment. Similar results have also been found in patients with stage III colon cancer diagnosed in Alberta [25,27]. Collectively, these results suggest that provision of free healthcare services does not, in and of itself, eliminate access barriers to standard care. Research is needed to improve understanding of barriers related to region/ neighborhood of residence and socioeconomic status so they can be addressed.

Few studies have compared standard treatment rates by disease stage for rectal cancer, but those that have also found lower adherence among patients with stage II disease relative to those with stage III disease [28]. Reasons for this are not clear, although it may be due, at least in part, to failure to refer patients to an oncologist. This is supported by a recent finding [29] that residents of Alberta diagnosed with stage II rectal cancer were less likely to have a consultation with an oncologist than those with stage III disease, a prerequisite to receiving radiation and/or chemotherapy in Alberta. Some surgeons and/or family physicians may not be aware of the difference in standard treatment for stage II rectal cancer versus stage II colon cancer. Alternatively, they may think that only tumors that have spread to lymph nodes (i.e., stage III) warrant adjuvant treatment. Regardless, efforts are needed to increase the proportion of rectal cancer patients who receive treatment consistent with guidelines in order to improve survival and maximize patient outcomes. Rectal cancer is a disease where treat- ment guidelines are continuously updated [30,31], therefore, ongoing discussions and education across medical specialists are especially important to ensure optimal treatment for patients.

Further investigation to identify the reasons for receiving delayed treatment is also needed to optimize patient outcomes. Physicians may delay radiation and/or chemotherapy due to a slow recovery from surgery, postsurgery complications, or possibly a change in the patient's decision to receive adjuvant therapy. Alternatively, delays may occur due to inefficiencies in the health care system or to shortages of resources to deliver care.

This is the first population-based study to investigate the association between timing of adjuvant treatment initiation and survival among patients with stage II/III rectal cancer. Current treatment guidelines in Alberta are supported by this study: patients with stage II/III rectal cancer should receive adjuvant treatment within 12 weeks of surgery. Patients who received adjuvant treatment $12-16$ weeks post-surgery or 16 weeks and more or did not receive it at all, were 1.43 and 1.58 times, respectively, more likely to die of rectal cancer than those who received treatment within 8 weeks of surgery, after adjusting for relevant factors. Although the HR for the group who received adjuvant treatment $12-16$ weeks after surgery was not statistically significant $(p=0.22)$, it is a clinically significant increase and is consistent with the HR for the " $16+$ weeks /no adjuvant chemotherapy" group. Furthermore, the rectal cancer-specific cumulative incidence curves for the two groups are similar to each other and distinct from the curves for the patients that were treated within 8 weeks or treated within 8 to 12 weeks of surgery. The statistical significance of this result was influenced by the relatively small number of patients who received adjuvant treatment $12-16$ weeks post-surgery (71 patients).

The finding that 12 weeks is the maximum time that should elapse from surgery to initiation of adjuvant treatment is consistent with findings from a similar study conducted on patients with stage III colon cancer in Alberta [19] as well as one that used the US National Cancer Institute's Surveillance, Epidemiology, and End Results data [32]. All three studies found that patients should receive adjuvant chemotherapy within 12 weeks of surgery to maximize patient survival. The consistency of these findings across different study populations, despite slightly different study methodologies, and across colon and rectal cancers is significant.

A surprising result was that patients who received neoadjuvant treatment had a significantly higher risk of both overall and rectal cancer-specific death than those who did not receive neoadjuvant treatment $(\mathrm{HR}=1.39$, $95 \%$ CI $1.05-1.84, \mathrm{p}=0.02$ and $\mathrm{HR}=1.52,95 \%$ CI $1.09-2.11, \mathrm{p}=0.01$, respectively). These results should 
be interpreted with caution. A probable explanation is that patients who received neoadjuvant treatment were different in clinically-relevant ways than those who did not receive it. Neoadjuvant treatment is given specifically to shrink tumors that are large and/or suspected of increasing the risk of local recurrence. Clinical trials have found neoadjuvant radiation and/or combined chemoradiation to be effective in doing so $[9,10]$, but no comparisons have been made between patients who are clinically determined to need neoadjuvant treatment and those who are not with respect to their risk of death. Our findings suggest that even though the risk of local recurrence is decreased amongst those who need and receive neoadjuvant treatment (based on clinical trials), their risk of rectal cancer-specific death is still higher than those patients who do not clinically require neoadjuvant treatment.

The findings of this study are robust and generalizable. Our linked dataset is a powerful tool and includes all patients diagnosed in the province of Alberta over a six-year period with up to nine years of follow-up. Only a clinical trial, however, can directly evaluate the effect timing of treatment has on survival. The intent of the study, however, was not to replace a clinical trial but rather to assess the association between treatment (and timing of it) and survival in a real clinical practice setting, in a population-based manner. Other limitations of the study, however, are that we did not have treatment details, such as completeness of the regimen or the specific treatment regimens received. Also, we did not have access to other clinical factors that could have an effect on patient survival, such as surgical complications, tumor grade, number of positive lymph nodes, and patient functional status.

In summary, our results support the importance of receiving adjuvant chemotherapy within 12 weeks of surgery as per current clinical treatment guidelines for patients with stage II or III rectal cancer. Only $43 \%$ of such patients diagnosed between 2000 and 2005 in Alberta, Canada, however, received the recommended treatment. Interventions are needed to ensure timely receipt of adjuvant chemotherapy for patients with stage II/III rectal cancer in order to optimize patient outcomes.

\section{Acknowledgements}

This research was made possible by grants received from the Canadian Institute for Health Research, Canadian Cancer Society, Alberta Cancer Foundation, Alberta Ingenuity Centre for Machine Learning, Alberta Innovates-Health Solutions, and Canada Research Chair Program. The authors thank Noha Sharaf El-Din for helping code adjuvant treatment and comments on the methodology and Angela Bella for her assistance in formatting the manuscript. The authors do not have any conflicts of interests or financial interests to disclose.

\section{REFERENCES}

[1] The National of Health Consensus Conference, "Adjuvant Therapy for Patients with Colon and Rectal Cancer," The Journal for American Medical Association, Vol. 264, No. 11, 1990, pp. 1444-1450. doi:10.1001/jama.1990.03450110090034

[2] H. O. Douglass Jr., C. G. Moertel, R. J. Mayer, et al., "Survival after Postoperative Combination Treatment of Rectal Cancer," The New England Journal of Medicine, Vol. 315, No. 20, 1986, pp. 1294-1295. doi:10.1056/NEJM198611133152014

[3] B. Fisher, N. Wolmark, H. Rockette, et al., "Postoperative Adjuvant Chemotherapy or Radiation Therapy for Rectal Cancer: Results from NSABP Protocol R-01," Journal of the National Cancer Institute, Vol. 80, No. 1, 1988, pp. 21-29. doi:10.1093/jnci/80.1.21

[4] J. E. Krook, C. G. Moertel, L. L. Gunderson, et al., "Effective Surgical Adjuvant Therapy for High-Risk Rectal Carcinoma," The New England Journal of Medicine, Vol. 324, No. 11, 1991, pp. 709-715. doi:10.1056/NEJM199103143241101

[5] Colorectal Cancer Collaborative Group, “Adjuvant Radiotherapy for Rectal Cancer: A Systematic Overview of 8507 Patients from 22 Randomized Trials," The Lancet, Vol. 358, No. 9290, 2001, pp. 1291-1304. doi:10.1016/S0140-6736(01)06409-1

[6] R. Sauer, H. Becker, W. Hohenberger, et al., "Preoperative Versus Postoperative Chemoradiotherapy for Rectal Cancer," The New England Journal of Medicine, Vol. 351, No. 17, 2004, pp. 1731-1740. doi:10.1056/NEJMoa040694

[7] L. L. Gunderson, D. J. Sargent, J. E. Tepper, et al., "Impact of T and N Stage and Treatment on Survival and Relapse in Adjuvant Rectal Cancer: A Pooled Analysis," Journal of Clinical Oncology, Vol. 22, No. 10, 2004, pp. 1785-1796. doi:10.1200/JCO.2004.08.173

[8] A. I. Neugut, A. T. Fleischauer, V. Sundararajan, et al., "Use of Adjuvant Chemotherapy and Radiation Therapy for Rectal Cancer among the Elderly: A Population-Based Study," Journal of Clinical Oncology, Vol. 20, No. 11, 2002, pp. 2643-2650. doi:10.1200/JCO.2002.08.062

[9] J. Folkesson, H. Birgisson, L. Pahlman, et al., "Swedish Rectal Cancer Trial: Long Lasting Benefits from Radiotherapy on Survival and Local Recurrence Rate," Journal of Clinical Oncology, Vol. 23, No. 24, 2005, pp. 5644-5650. doi:10.1200/JCO.2005.08.144

[10] M. S. Roh, L. H. Colangelo, M. J. O'Connell, et al., "Preoperative Multimodality Therapy Improves Disease-free Survival in Patients with Carcinoma of the Rectum: NSABP R-03," Journal of Clinical Oncology, Vol. 27, No. 31, 2009, pp. 5124-5130. doi:10.1200/JCO.2009.22.0467

[11] National Comprehensive Cancer Network, "Rectal Cancer V.2. NCCN Clinical Practical Guidelines in Oncology $2010, " 2010$.

http://www.nccn.org/professionals/physician_gls/PDF/rec tal.pdf

[12] B. Glimelius, J. Oliveira and ESMO Guidelines Working 
Group. "Rectal Cancer: ESMO Clinical Recommendations for Diagnosis, Treatment and Follow-Up," Annals of Oncology, Vol. 20, No. S4, 2009, pp. 54-56. doi:10.1093/annonc/mdp128

[13] T. C. Tucker, H. L. Howe and H. K. Weir, "Certification for Population-Based Cancer Registries," Journal of Registry Management, Vol. 26, No. 1, 1999, pp. 24-27.

[14] A. Fritz, C. Percy, A. Jack, et al., "International Classification of Diseases for Oncology," World Health Organization, Geneva, 2000.

http://www.who.int/classifications/icd/adaptations/oncolo gy/en/index.html

[15] F. L. Greene, D. L. Page, I. D. Fleming, et al., "AJCC Cancer Staging Manual 6th Edition," Springer-Verlag, New York, 2002. http://www.cancerstaging.org/products/ajccproducts.html

[16] World Health Organization, "Statistical Classification of Diseases and Related Health Problems10th Revision. v.2 Instruction Manual," World Health Organization, Geneva, 1994, pp. 30-85.

[17] H. Quan, V. Sundararajan, P. Halfon, et al., "Coding Algorithms for Fefining Comorbidities in ICD-9-CM and ICD-10 Administrative Data," Medical Care, Vol. 43, No. 11, 2005, pp. 1130-1139. doi:10.1097/01.mlr.0000182534.19832.83

[18] W. D'Hoore, C. Sicotte and C. Tilquin, "Risk Adjustment in Outcome Assessment: The Charlson Comorbidity Index," Methods of Information Medicine, Vol. 32, No. 5, 1993, pp. 382-387.

http://europepmc.org/abstract/MED/8295545

[19] Lima, Y. Yasui, S. Andrew, et al., "Association between Receipt and Timing of Adjuvant Chemotherapy and Survival for Patients with Stage III Colon Cancer in Alberta, Canada," Cancer, Vol. 117, No. 16, 2011, pp. 3833-3840. doi:10.1002/cncr.25954

[20] T. A. Gooley, W. Leisenring, J. Crowley, et al., "Estimation of Failure Probabilities in the Presence of Competing Risks: New Representations of Old Estimators," Statistics in Medicine, Vol. 18, No. 6, 1999, pp. 695-706. doi:10.1002/(SICI)1097-0258(19990330)18:6<695::AID$\underline{\mathrm{SIM} 60>3.0 . \mathrm{CO} ; 2-\mathrm{O}}$

[21] S. Durrleman and R. Simon, "Flexible Regression Models with Cubic Splines," Statistics in Medicine, Vol. 8, No. 5, 1989, pp. 551-561. doi:10.1002/sim.4780080504

[22] R. K. Wong, S. Berry, K. Spithoff, et al., "Preoperative or Postoperative Therapy for Stage II or III Rectal Cancer: An Updated Practice Guideline," Clinical Oncology, Vol. 22, No. 4, 2010, pp. 265-271. http://www.ncbi.nlm.nih.gov/pubmed/20398849
[23] T. J. Iwashyna and E. B. Lamont, "Effectiveness of Adjuvant Fluorouracil in Clinical Practice: A PopulationBased Cohort Study of Elderly Patients with Stage III Colon Cancer," Journal of Clinical Oncology, Vol. 20, No. 19, 2002, pp. 3992-3998. doi:10.1200/JCO.2002.03.083

[24] V. Sundararajan, N. Mitra, J. S. Jacobson, et al., "Survival Associated with 5-Fluorouracil-Based Adjuvant Chemotherapy among Elderly Patients with Node-Positive Colon Cancer," Annals of International Medicine, Vol. 136, No. 5, 2002, pp. 349-357.

http://www.ncbi.nlm.nih.gov/pubmed/11874307

[25] D. J. Sargent, R. M. Goldberg, S. D. Jacobson, et al., “A Pooled Analysis of Adjuvant Chemotherapy for Resected Colon Cancer in Elderly Patients," The New England Journal of Medicine, Vol. 345, No. 15, 2001, pp. 1091-1097. doi:10.1056/NEJMoa010957

[26] I. H. Zuckerman, T. Rapp, E. Onukwugha, et al., "Effect of Age on Survival Benefit of Adjuvant Chemotherapy in Elderly Patients with Stage III Colon Cancer," Journal of American Geriatrics Society, Vol. 57, No. 8, 2009, pp. 1403-1410. http://www.ncbi.nlm.nih.gov/pubmed/19563521

[27] M. Winget, S. Hossain, Y. Yasui, et al., "Characteristics of Stage III Colon Adenocarcinoma Patients Who Fail to Receive Guideline-Recommended Treatment," Cancer, Vol. 116, No. 20, 2010, pp. 4849-4856. doi:10.1002/cncr.25250

[28] J. Z. Ayanian, A. M. Zaslavsky, C. S. Fuchs, et al., "Use of Adjuvant Chemotherapy and Radiation Therapy for Colorectal Cancer in a Population-Based Cohort," Journal of Clinical Oncology, Vol. 21, No. 7, 2003, pp. 1293 1300. doi:10.1200/JCO.2003.06.178

[29] N. S. Eldin, Y. Yasui, A. Scarfe, et al., "Adherence to Treatment Guidelines in Stage II/III Rectal Cancer in Alberta, Canada," Clinical Oncology, Vol. 24, No. 1, 2012, pp. e9-e17. doi:10.1016/j.clon.2011.07.005

[30] V. Valentini, C. Aristei, B. Glimelius, et al., "Multidisciplinary Rectal Cancer Management: 2nd European Rectal Cancer Consensus Conference (EURECACC2)," Radiotherapy \& Oncology, Vol. 92, No. 2, 2009, pp. 148-163. doi:10.1016/j.radonc.2009.06.027

[31] Alberta Health Services, "Clinical Practice Guideline GI-005: Early Stage Rectal Cancer,” 2010. http://www.albertahealthservices.ca/hp/if-hp-cancer-guide -gi005-early-stage-rectal.pdf

[32] D. Hershman, M. J. Hall, X. Wang, et al., "Timing of Adjuvant Chemotherapy Initiation after Surgery for Stage III Colon Cancer," Cancer, Vol. 107, No. 11, 2006, pp. 2581-2588. doi:10.1002/cncr.22316 